\title{
MALEVOLENT FILE DETECTION IN SHORT RANGE COMMUNICATION
}

\author{
Saranya Devi B ${ }^{1}$, Maragadham ${ }^{2}$
}

\begin{abstract}
Short Range communication likely termed as Delay Tolerance Network in Network design is presented whichaddresses issues in non-homogeneous system wouldreduceconstantsystemaccessibility. Proximity malevolent is a malware that goes into networks by the means of Bluetooth, Wi-Fi and so forth and adventures the opportunistic contacts for transmission. The Behavioral portrayal of malevolent is an alternative approach to deal with recognising proximity malevolent. There being a danger with choice in behavioural malevolentportrayal, extension named Look Ahead is proposed. Moreover, two augmentations are created, filtering and Versatile Look Ahead to expel the test of malevolent hubs sharing false confirmations. In the proposed research, extension to Naïve Bayes approach for malevolent detection is presented to address the test of "Malevolent node sharing false confirmations" accomplishing high recognition rate than past methodologies.
\end{abstract}

Keywords: Versatilelook Ahead, Proximity Malevolent, Malevolent Detection, Filtering,

\section{INTRODUCTION}

The Internet relies on upon consistent end-to-end network and dependability for affirmations. Nature and mischance effortlessly break the end-to-end network. Bundles that can't be transmitted because of this breakage can be effectively disposed of. DTNs utilize steering nodes with the capacity to protect the information's the point at which the linkage is down. Information packs are put away until the following bounce is restored, then they are sent on, which implies end destination need not be consistently associated.The storage capacity of the messages is indefinite. DTN empowers programmed information correspondence administration in short range communication. DTN is utilized as a part of numerous situations where the systems have long deferred or disturbance e.g Military Network, space and so on. DTN gives solid information exchange, retransmission from nearest node rather from the sender. Because of which DTNs are vulnerable towards malware attacks.

Malware spread concerns parasitic programming sections that join themselves to some existing executable substance. The section might be machine code that infects some existing application, utility, or framework program, or even the code used to boot a PC system. Malware is characterized by its maliciousintent, acting against the prerequisites of the PC client, and does exclude programming that causes harmnot intentionally because of some deficiency. The term badware is infrequently utilized, and connected to both genuine malware and unexpectedly harmful programming.

The best-known sorts of malware, infections and worms, are known for the way in which they spread, as opposed to a particular sorts of conduct. The term PC infection is utilized for a project that installs itself in some other executable programming on the objective framework without the client's assent and when that is run causes the infection to spread to different executables. Then again, a worm is a stand-alone malware program that effectively transmits itselfcontaminates different PCs. These definitions lead to the perception that an infection requires the client to run aninfected program or working framework for the infection to spread, while a worm spreads itself.

Malware uses pair-wise communication mechanisms such as Bluetooth to spread. The common way that malware uses to propagate is to exploit the vulnerabilities in the Bluetooth communication stack.For e.g, the famous worm Cabir worm that proliferates over Bluetooth utilizing caribe.sis package.

The popularity of the electronics devices, like laptops, smartphones and so on, resuscitates the delay-tolerancenetwork model as a substitute to traditional infrastructural model. The wide acceptance of these gadgets, combined with strong commercial inducements, incites a class of malware that centers DTNs. This class of malware is termed as Proximity Malevolent. A precondition to protecting against proximity malevolent is to identify it. In this paper, we study a commonconductportrayal of proximity malevolent. Malware contaminated nodes seen amid their sharp meets:Individual perception could be defective; yet unusual practices of tainted hubs are identifiable over the long haul.

\section{RELATED AND EXISTING WORK}

Network is group of Nodes. Each node will connect with its neighbors and share their information. On the off chance that a node is influenced by a malware it's important to clear 
it else its neighbors will connect with it and they additionally get infected by malware. Hencedetecting malware is necessary.

With the acceptance of new short range communication technologies like Wi-Fi and Near field communication (NFC) which exchanges mass information exchange between vicinity gadgets, the risk of proximitymalevolent is turning out to be more practical. Proximity malware in light of the DTN model brings security challenges that are not introduced in the model. There are many techniques used to encounter the malware attacks.

"Distributed Malevolent detection based on binary file": ML (Machine learning) procedures plays a vital role in detecting the malware. This is based on the feature extraction on binary file to the image projection faces a challenge of growing array.

"Behavior-Based Malware Analysis and Detection": Examinations are made on the extraction of malevolent conduct and the formal Malevolent Behavior Feature (MBF) extraction methodology. In this way a malevolent conduct based malevolentexposure program is proposed. The use of this strategy shows that it can perceive as of late framed dark malevolent.

\section{PROBLEM STATEMENT}

DTN specific malware associated harms are:

- Insufficient evidence vs. evidence collection risk. The evidence is collected only when the nodes communicate with each other. But communicating with the infested nodes carries the danger of getting contaminated.

- Filtering false evidence. Sharing the evidences among the opportunistic contacts helps us to solve the insufficient evidence issue, but sharing false evidence between nodes leads to negate the benefits of sharing.

- Liars. Sharing false appraisal between nodes to befuddle others is finished by the evil nodes. A false appraisal could be a false acclaim or a false allegation.Additionally, false evaluations on a node are given by the liar node with whom it has not by any means met.

- Defectors. Changing the nature of the node due to malware infection. These start as a good node however get to beshrewd because of malware disease by sharing evaluations.

When we transmit the infected document which contains virus, worm etc, being advantage to harm the devices in the system. We distinguish these infected documents and limit them from transmission and give the Quality of Service to clients.
Albeit various plans have been proposed to shield against malevolent assaults on the Internet and in remote systems, they accept industrious network and can't be specifically connected to DTNs that have discontinuous availability. Subsequently, it is still an open issue to address infuse assaults in DTNs.

\section{PROPOSED SYSTEM}

Protecting the network from malevolent traffic is a difficult issue that requires coordination of many components which includes technical and non-technical solutions. Therefore, implementing malevolent detection is a tedious task due to the network with many vulnerabilities and security issues.

The problem statement stated above is been solved by our proposed system filtering and Versatile look ahead.

A. Filtering: In light of the perception of one's own appraisals are honest, which can be utilized to bootstrap the confirmation hardening process.

B. Versatile look ahead: Thistakes an alternate methodology toward evidence solidification. Rather than choosing whether to utilize the evidencegave by others specifically in the cut-off choice, versatile look ahead in a roundabout way utilizes the evidenceby adjusting the progressions to look ahead to the differing qualities of assessment.

\section{DESIGN}

Consider a DTN including $\mathrm{n}$ nodes. The neighbors of a node are the nodes it has (entrepreneurial) contact opportunities with. Proximitymalevolent is a noxiousframework that disappointmentsthat miracles the host nodes commonplace limit and has a fix of duplicating itself to various nodes in the midst of (sharp) contact opportunities between nodes in the DTN. Exactly when duplication happens, the other hub is debased with the malware. In our model, we expect that each hub is fit for assessing the other party for suspicious exercises after every experience.

On the off chance that node $\mathrm{i}$ has $\mathrm{N}$ (pairwise) encounters with its neighbors and $\mathrm{sN}$ of them are reviewedas doubtful by the neighbors, its dishonesty $\mathrm{Si}$ is characterized as the proof gave by others specifically in the cut-off choice, versatile look ahead by implication utilizes the proof by $\mathbf{S i}=\lim \mathbf{s N}$

By calculating suspiciousness value $\mathrm{Si}$, we judge a node whether it is good or evil. Rather than expecting a refined malware method for dealing with stress, 


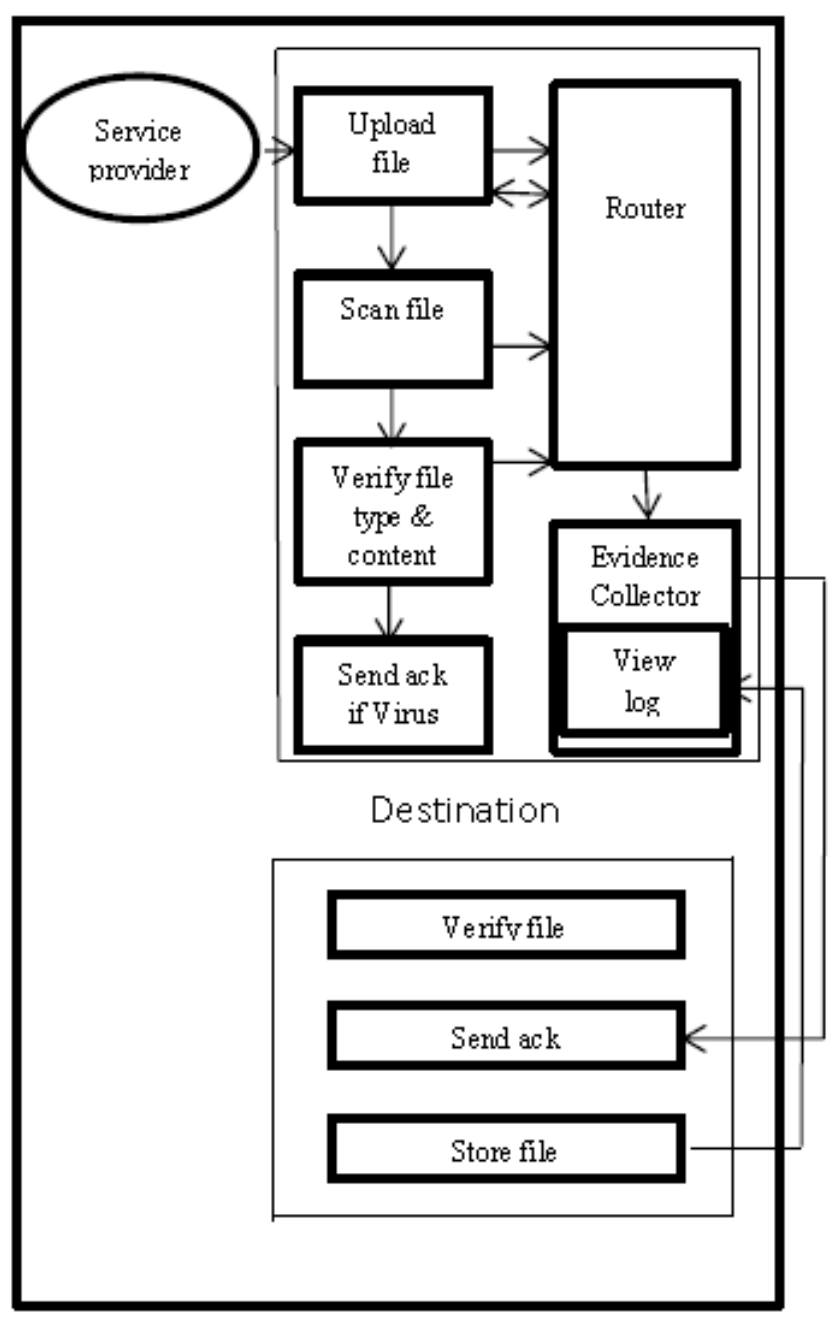

Fig. 1 System Architecture

for example, fixing or self-mending, we consider a basicwhat's more, generally pertinent malware regulation technique: Taking into account past assessments, a centre point I picks whether to decrease future affiliations (cut off) with a neighbor $\mathrm{j}$.

\section{CONCLUSIONS}

We exhibit look ahead, alongside Filtering and versatile look ahead, to address two noveldifficulties in DTNs: inadequate proof versus proof gathering hazard and separating false confirmation.

\section{ACKNOWLEDGMENT}

Proposed work is a job of great enormity and it can't be accomplished by an individual all by them. Eventually, I am very much grateful to the Vice Principal and Head of the Department Dr. R.J. Anandhi for her unfailing encouragement and suggestion given to me in the course of my project work.I convey my sincere gratitude to my guide Mrs. Maragadham, Assistant professor, Department of CSE, for having constantly guided and monitored the development of the project work.

A note of thanks to the Department of Computer Science Engineering, both teaching and non-teaching staff for their co-operation extended to us.I thank my parents for their constant support and encouragement. Last, but not the least, I would like to thank my peers and friends.

\section{REFERENCES}

[1]. Xiaoguang Han ; Jigang Sun ; Wu Qu ; Xuanxia Yao (2014). Distributed malware detection based on binary file features in cloud computing environment.

[2]. Liu Wu ; Ren Ping; Liu Ke; Duan Hai-xin(2011). Behaviour-Based Malware Analysis and Detection.

[3]. R. Villamarı'n-Salomo'n and J. Brustoloni,(2013) "Bayesian Bot Detection Based on DNS Traffic Similarity," Proc.

[4]. Trend Micro Inc. (2004) SYMBOS CABIR.A. [Online]. Available: http://goo.gl/aHcES

[5]. NFC Forum. About NFC. [Online]. Available: http://goo.gl/zSJqb

[6]. Wi-Fi Alliance. Wi-Fi Direct. [Online]. Available: http://goo.gl/fZuyE

[7]. Kolbitsch, P. Comparetti, C. Kruegel, E. Kirda, X. Zhou, and X. Wang, "Effective and efficient malware detection at the end host," in Proc. USENIX Security, 2009.

[8]. U. Bayer, P. Comparetti, C. Hlauschek, C. Kruegel, and E. Kirda, "Scalable, behavior-based malware clustering," in Proc. IEEE NDSS, 2009.

[9]. Behavioral Malware Detection in Delay Tolerant Networks, Wei Peng, Student Member, IEEE, Feng Li,Member, IEEE, Xukai Zou, Member, IEEE, and Jie Wu, Fellow, IEEE 